\title{
Towards a quantitative comparison between global and local stability analysis
}

\author{
L. Siconolfi ${ }^{1} \dagger$, V. Citro ${ }^{2}$, F. Giannetti ${ }^{2}$, S. Camarri ${ }^{1}$, P. Luchini ${ }^{2}$ \\ ${ }^{1}$ DICI, Università di Pisa, Via Girolamo Caruso, 56122 Pisa, Italy \\ ${ }^{2}$ DIIN, Universitá degli Studi di Salerno, Via Giovanni Paolo II, 84084 Fisciano (SA), Italy
}

(Received $\mathrm{xx}$; revised $\mathrm{xx}$; accepted $\mathrm{xx}$ )

We propose a methodology to estimate with reasonable accuracy the characteristics of bluff-body wakes by local stability analysis under the assumption of weakly non-parallel flows. Firstly, a generalization of the classical spatio-temporal stability analysis is shown and applied to a fully 3D flow. Secondly, a higher-order correction term to the classical saddle-point analysis is included in the analysis, showing that this correction is crucial to reach a quantitatively good agreement between local and global stability analyses. Moreover, the included correction is also shown to lead to a definitely improved prediction of the spatial structure of the identified globally unstable mode by means of local analysis, which is definitely less demanding than global analysis in term of computational costs. Such procedure is first validated for the case of the flow past a circular cylinder and then applied to the fully three-dimensional wake flow past a sphere. Both the estimated unstable eigenvalue and the associated direct-mode structure are determined from local stability properties and compared with results obtained from a full three-dimensional global stability analysis. Results show excellent agreement between the numerical global solution when the correction term is taken into account.

\section{Introduction}

Spatially developing open flows such as bluff-body wakes or jets may display a natural unsteady instrinc dynamics (self-sustained oscillations or Global modes), where the flow behaves like a global oscillators. For these flows, the fundamental concept of local absolute frequency $\omega_{0}$ has been introducted in order to investigate their dynamical behaviour (Briggs (1964),Bers (1983)). In the regions of absolute instability, i.e. $\omega_{0, i} \equiv \operatorname{Im}\left(\omega_{0}\right)>0$, the perturbations are not convected downstream and grow in place driving the evolution of the nonlinear dynamical system. When a sufficient large region of absolute instability develops, a self-sustained resonances occurred as the results of the global stability analysis. (see Chomaz et al. (1988), Huerre \& Monkewitz (1985) and Monkewitz et al. (1993)).

In this context, the dynamics of the perturbations can be analyzed both from a local or a global point of view. In the local analysis, under the assumption of weakly non-parallel flows, the perturbations are considered as the superimposition of normal waves on the base-flow at each streamwise station. On the other hand, in the global approach the time evolution of perturbations in the whole physical domain is investigated. In the past, several works have been devoted to investigate the connection between the global stability features and the local ones for different flow configurations $(* * *)$. For instance, in the review by Huerre \& Monkewitz (1990) and in Huerre \& Rossi (1998), the occurrence of an unstable global mode in terms of the local properties of the flow field is dealt with

$\dagger$ Email address for correspondence: lorenzo.siconolfi@for.unipi.it 
the classical WKBJ asymptotic analysis (Bender \& Orszag 1978). Particular attention was devoted to the role of absolute-instability region and to the identification, in the context of the local analysis, of the complex spatial position where the flow is most sensitive to change in internal feedback mechanism, usually referred as wavemaker. The possibility to perform a global stability analysis by using a local approach leads to a substantial saving of the computational time and of the required computer resources, since the global eigenvalue problem related to the discretization of the stability problem on the whole domain is recast into a several small eigenvalue problems. However, the use of the WKBJ approximation can be justified for base flows that evolve slowly in space in comparison with the typical instability wavelength. Nevertheless, this approximation has been successfully applied beyond the limit of its validity. For example, Giannetti \& Luchini (2007) showed a comparison between the linear global and local stability analysis for the case of the flow past a circular cylinder, for Reynolds numbers $(R e)$ ranging from 5 to 100 (based on the incoming velocity and diameter of the cylinder). Subsequently, in Pier (2008) the flow past a fixed sphere is considered, where the local stability features are obtained under a quasi-parallel flow assumption. Finally, Juniper \& Pier (2015) showed that a local analysis can be used also for the identification of the adjoint modes and the structural sensitivity, showing the results for a slowly-developing confined wake and for the flow past a circular cylinder at $\mathrm{Re}=50$. Unfortunately, in all of these studies, the resulting eigenvalues computed by a local approach can be only used for qualitative analyses and, in general, the inherent errors in comparison with to the reference global solutions (growth rate, frequency and mode structure) can be significant.

In this context, our aim is to show that by including a higher-order correction term of the WKBJ approximation, the local approach can be used to provide a quantitative estimation of the global eigenvalue and spatial characteristics of the corresponding mode. Furthermore, we also show that a two-dimensional local analysis can be used to study fully three-dimensional wakes. We underline that such two-dimensional local approach is orders of magnitude cheaper than the global three-dimensional modal analysis.

The paper is organized as follows. In section 2 the basic concepts of the global and local are recalled, together with the main results of the WKBJ approximation. The numerical techniques used in this paper are briefly described in section 3 , while in section 4 the main results are presented in details.

\section{Theoretical framework}

Let us consider a three-dimensional flow of a viscous fluid described by the unsteady incompressible Navier-Stokes equations

$$
\partial_{t} \mathbf{u}+\mathbf{u} \cdot \nabla \mathbf{u}+\nabla p-R e^{-1} \nabla^{2} \mathbf{u}=0 \quad \text { and } \quad \nabla \cdot \mathbf{u}=0
$$

where $\mathbf{u}=(u, v, w)$ is the velocity field, $p$ is the reduced pressure and $R e=U \ell \nu^{-1}$ is the flow Reynolds number based on the reference length $\ell$, the reference velocity $U$ and the kinematic viscosity $\nu$. The system (2.1) is completed by appropriate boundary conditions. The investigation of the instability mechanism acting in a given flow relies on the determination of a stationary solution $\boldsymbol{Q}_{\boldsymbol{b}}=\left[\boldsymbol{u}_{b}, P_{b}\right]$ of the system (2.1), usually referred as base flow. Depending on the characteristics of the base flow, different stability approaches can be used to investigate the behaviour of the fluid flow (see Theofilis (2011) for a review). 


\subsection{The global stability analysis}

When no assumptions can be made on the base flow, a global stability analysis has to be considered in order to take into account the three-dimensional flow inhomogeneities. Here, the long-term stability properties are studied by considering the asymptotic behaviour of a small-amplitude perturbation $\boldsymbol{q}^{\prime}=\left[\boldsymbol{u}^{\prime}, P^{\prime}\right](x, y, z, t)$ superimposed on the base flow $\boldsymbol{Q}_{\boldsymbol{b}}$ and sought in the classical normal mode form $\boldsymbol{q}^{\prime}=\hat{\boldsymbol{q}}(x, y, z) \exp \left\{-i \omega_{G} t\right\}$. Introducing this decomposition and the normal mode ansatz in (2.1), a stationary problem for the base flow field and a global stability eigenvalue problem for the perturbation are found. Considering the size of the problem, a time-stepper procedure involving the linearized Navier-Stokes operator $\mathcal{L}$ is usually adopted in order to solve the stability problem. The resulting system can be recast as follows:

$$
\partial_{t} \mathbf{q}^{\prime}+\mathcal{L}\left(\mathbf{Q}_{\mathbf{b}}, R e\right) \mathbf{q}^{\prime}=0 .
$$

When the system (2.2) is supplemented by appropriate homogeneous boundary conditions and spatially discretized, a generalized eigenvalue problem is found. Although this approach seems more convenient for its general application, the characteristic sizes of the involved discrete problems require high computational resources to be solved.

In order to circumvent this problem, some assumptions on the fluid flow can be considered to study the nature of the instability mechanisms with lower computational costs.

\subsection{The local stability analysis}

When the base flow, described here in Cartesian coordinates $(x, y, z)$, slowly evolves along a given direction, (assumed to be aligned with the $x$ axis), a local linear stability analysis can be performed, as a generalization of the classical Orr-Sommerfled/Squire theory. In the spirit of the local stability analysis, the base flow is considered to depend only on the two direction $(y, z)$ and independent on the streamwise $x$-direction, i.e. $\boldsymbol{Q}_{\boldsymbol{b}}=\left[\boldsymbol{u}_{b}, P_{b}\right](y, z)$. In such cases, a small-amplitude perturbation $\boldsymbol{q}^{\prime}$ of the base flow can be searched in the following modal form:

$$
\boldsymbol{q}^{\prime}=\left[\boldsymbol{u}^{\prime}, P^{\prime}\right](x, y, z, t)=\hat{\boldsymbol{q}}(y, z) \exp \left\{i\left(k x-\omega_{L} t\right)\right\},
$$

where $k$ is the streamwise complex wavenumber and $\omega_{L}$ is the local complex eigenvalue. The perturbations are then three-dimensional, but a sinusoidal shape is considered in $x$-direction. When the modal form 2.3 is substituted in 2.1 and linearized the equations around the base flow, an eigenfunction problem is obtained. Because of the local assumption, the coefficients of the resulting stability problem are independent on the $x$-direction, i.e. $\partial_{x} \boldsymbol{Q}_{\boldsymbol{b}}=0$. Thus, once completed the mathematical problem with the appropriate boundary conditions and discretized using a generic numerical method, the stability problem can be formally recast as follows:

$$
\left(\boldsymbol{A}_{\mathbf{0}}+\boldsymbol{A}_{\mathbf{1}} k+\boldsymbol{A}_{\mathbf{2}} k^{2}\right) \hat{\boldsymbol{q}}=\omega_{L} \boldsymbol{B} \hat{\boldsymbol{q}},
$$

where $\boldsymbol{A}_{\mathbf{0}}, \boldsymbol{A}_{\mathbf{1}}, \boldsymbol{A}_{\mathbf{2}}, \boldsymbol{B}$ are complex matrices given by the discretization of the linearized Navier-Stokes equations (for further details see Siconolfi et al. (2015)). Starting from 2.4, different generalized eigenvalue problems occur, depending on the type of the analysis. The linear temporal analysis is carried out considering the wavenumber $k \in \operatorname{Re}$ as parameter, while the eigenvalue $\omega_{L} \in \operatorname{Im}$ is shouted, where the real part, i.e. $\operatorname{Re}\left(\omega_{L}\right)$, is the angular velocity and the imaginary part, i.e. $\operatorname{Im}\left(\omega_{L}\right)$, is the temporal growth rate. In the quadratic spatial analysis, otherwise, the frequency $\omega_{L} \in$ Re is the parameter and the complex wavenumber $k$ is shouted, where its real part, i.e. $\operatorname{Re}(k)$, is related with the 
periodicity length in the homogeneous $x$-direction and the imaginary part, i.e. $\operatorname{Im}(k)$, is the corresponding spatial growth rate.

\subsection{The evaluation of the linear global mode from the local analysis}

A connection between global and local instability characteristics is available using a WKBJ-type analysis when the base flow can be considered weakly non-parallel, i.e. the ratio $\epsilon$ between the characteristic instability wavelength $\lambda$ and the characteristic spatial length scale $L$ of the base flow is small $(\epsilon \ll 1)$ (Huerre \& Monkewitz 1990). Thus a new slow space scale $X \equiv \epsilon x$ can be introduced and the dispersion relation is then recovered by freezing the new $X$-coordinate: $D[k, \omega ; X, R e]=0$ (in the following the dependence from $R e$ is omitted for brevity).

First of all, the saddle point of this dispersion relation in the complex $k$-plane at each $X$ streamwise station identifies the absolute complex eigenvalue $\omega_{0}(X)=\omega\left(k_{0} ; X\right)$, such that $\partial \omega / \partial k\left(k_{0} ; X\right)=0$. The existence of a finite region where the absolute growth rate $\omega_{0, i}>0$, i.e. $\omega_{0, i}=\operatorname{Im}\left(\omega_{0}(X)\right)$, is a necessary condition for the onset of selfsustained global oscillations (Chomaz et al. 1988). Considering a double infinite flow domain and under the assumption of the regularity of the $D[k, \omega ; X]=0$, the unknown global eigenvalue $\omega_{G}$ can be written as (Monkewitz et al. 1993):

$$
\omega_{G}=\omega_{s}+\epsilon \omega_{\epsilon} \quad \text { with : } \omega_{s}=\omega_{0}\left(X_{s}\right)
$$

where $X_{s}$ is the complex saddle point, or second-order turning point, such that $\partial \omega_{0} / \partial X\left(X_{s}\right)=0$ and $\epsilon \omega_{\epsilon}$ is a small high-order correction term. Once $\omega_{G}$ has been identified at leading order, thus neglecting the term $\epsilon \omega_{\epsilon}$, the distribution of the direct global mode $\hat{\boldsymbol{q}}(x, y, z)$ can be expressed, in the region away from the turning point $X_{s}$, as:

$$
\hat{\boldsymbol{q}}(x, y, z) \sim A^{\mp}(X) \hat{\boldsymbol{q}}(y, z ; X) \exp \left[i \epsilon^{-1} \int_{X_{s}}^{X} k^{\mp}\left(\omega_{s} ; X^{\prime}\right) d X^{\prime}\right]
$$

where $A(X)$ is a slowly evolving amplitude, $\hat{\boldsymbol{q}}(y, z ; X)$ is the local eigenfunction of problem (2.4) and the superscripts ${ }^{-}$and ${ }^{+}$refer respectively to the region upstream and downstream of the saddle point $X_{s}$.

The identification of the frequency of the self-sustained flow oscillations is one of the main objective of a global stability analysis. In order to obtain an accurate estimation of the global eigenvalue, the correction term $\epsilon \omega_{\epsilon}$ in (2.5) can be also considered.

In a small region of size $O\left(\epsilon^{1 / 2}\right)$ around the turning point $X_{s}$, the WKBJ approximation breaks down. If a new inner streamwise scaling $\bar{X} \equiv\left(X-X_{s}\right) \epsilon^{-1 / 2}$ is considered, the perturbation in the region near $X_{s}$ can be sought as follows:

$$
\overline{\boldsymbol{q}} \sim \bar{A}(\bar{X}) \exp \left[i \epsilon^{-1} k_{0, s}\left(X-X_{s}\right)\right] \quad \text { with : } k_{0, s}=k_{0}\left(X_{s}\right)
$$

where the $\bar{A}(\bar{X})$ is found by imposing a suitable orthogonality condition (Fredholm alternative) at higher-order (see Huerre \& Monkewitz (1990)). Finally, the eigenvalue correction term $\omega_{\epsilon}$ is evaluated by the asymptotic matching of the outer WKBJ solution with that in the inner double turning point region. In particular, $\omega_{\epsilon}$ is restricted to assume discrete values such that (Huerre \& Monkewitz 1990):

$$
\omega_{G} \sim \omega_{s}+\epsilon \omega_{\epsilon} \quad \text { with } \quad \omega_{\epsilon}=\left[\delta \omega-\frac{i}{2} \omega_{k k} k_{0 X}+\sqrt{\omega_{0 X X} \omega_{k k}}\left(n+\frac{1}{2}\right)\right],
$$

where $n$ is an integer and $\delta \omega$ takes into account truly non-local effects. All the quantities $\omega_{k k}=\partial^{2} \omega / \partial k^{2}, k_{0 X}=\partial k_{0} / \partial X, \omega_{0 X X}=\partial^{2} \omega_{0} / \partial X^{2}$ are evaluated at the complex saddle point $X_{s}$. Except for the constant $\delta \omega$, all the quantities involved in (??) can be extracted 
from the behavior of the dispersion relation around $X_{s}$ and $k_{0}$.

In the following, the complex global eigenvalue from the global stability analysis is labelled $\omega_{G}(g l o b)$. The global eigenvalue evaluated from the WKBJ approximation at order $O\left(\epsilon^{0}\right)$, equivalent to the saddle point condition, is labelled $\omega_{G, 0}(l o c)$, i.e. $\omega_{G, 0}(l o c)=$ $\omega_{s}$, and the value at order $O\left(\epsilon^{1}\right)$ is designed $\omega_{G, 1}(l o c)$, i.e. $\omega_{G, 1}(l o c)=\omega_{s}+\epsilon \omega_{\epsilon}$. Finally, the expressions without correction and with correction will be used in the body of the text for the quantities evaluated considering $\omega_{G, 0}(l o c)$ and $\omega_{G, 1}(l o c)$, respectively.

\section{Numerical approach}

DNS simulations documented here are carried out using Nek5000 (http://nek5000. mcs.anl.gov), which is a spectral-element code based on Lagrange polynomial interpolants in the $P_{N}-P_{N-2}$ formulation, based on tensor-product arrays of Gauss-LobattoLegendre (GLL) quadrature points in each hexahedral element. A third-order backward differentiation formula is used for the time discretization. The viscous terms are treated implicitly while an explicit scheme in time is considered for the non-linear convective ones by a third-order forward extrapolation. In global stability analysis, the linearized direct and adjoint Navier-Stokes equations for a small-amplitude disturbance is solved using Nek5000. The code is used as a time-stepper and it is coupled with the IRAM eigenvalue solver implemented in ParPACK. For the local stability analysis, spatial discretization is obtained by the Taylor-Hood finite element method using the code FreeFem++ (http: //www.freefem.org). Both the resulting temporal and the spatial stability problems are solved by a Krylov-Schur method with a shift-invert technique, using the implementation already available in the SLEPc library (http://slepc.upv.es). Higher order WKBJ correction terms can be included to increase the accuracy of the approximation. In particular the eigenvalue drift $\omega_{\epsilon}$ can be computed by evaluating the expression in square brackets in formula (2.8). Particular attention must be paid when evaluating such terms. Indeed, the determination of the complex saddle point and the calculation of the other terms appearing in (2.8) must be performed by analytic continuation of the dispersion relation in the complex $X$ plane. Such procedure is generally performed by fitting the available data with a simple polynomial (Pier 2002) or rational function (Cooper \& Crighton 2000) and successively by evaluating the interpolant for complex $X$ values. While the leading order term $\omega_{s}$ has been evaluated without problems by several authors by using different procedures, the choice of an appropriate interpolation/extrapolation technique becomes crucial to accurately evaluate the correction $\omega_{\epsilon}$. Such term is in fact small compared to $\omega_{s}$ so that special attention must be paid in selecting a procedure that minimizes high-frequency errors. The presence of such components, in fact, can lead to errors even larger than the correction itself. In this paper, we adopted a particularly accurate reconstruction procedure. First, the data available for real $X$ value are fitted using cubic splines in order to smooth out the high-frequency error terms. Secondly, Chebyshev rational approximation is used for the function reconstruction in the complex $X$ plane as implemented in the Matlab library Chebfun (http://www.chebfun.org). Such procedure allows for an accurate determination of the global eigenvalue $\omega_{G}$. The spatial evolution of the global mode structure can be determined by inspecting equation (2.6). Non-parallel effects are accounted for by the amplitude function $A(X)$ which can be determined by solving the compatibility equation for the second order WKBJ approximation. Another possibility, which is equivalent (up to higher order terms) is to evaluate the eigenfunction at every streamwise location using the corrected value $\omega_{G}$ in place of the saddle point eigenvalue $\omega_{s}$ : in this way it is possible to include the amplitude 
term directly in the eigenfunction shape. Such procedure has been adopted in this paper to accurately reconstruct the direct global mode.

\section{Results}

In this section, we show the results obtained in estimating the global stability properties of a globally unstable flow using the local stability analysis as detailed in sections 2 . To this purpose, we consider three different flow configurations: the two-dimensional flow past a circular cylinder, the laminar boundary layer (BL) flow over a hemispherical roughness element placed on a flat plate and the flow past a fixed sphere invested by a uniform stream. The flow past a circular cylinder is a well documented case in the literature and, for this reason, it also provides us with a validation of the used tools. Conversely, the other two cases are complex fully three-dimensional configurations. Results pertaining to the flow configurations are discussed in the following sections.

\subsection{Flow past a circular cylinder}

The two-dimensional flow past a circular cylinder is considered here. All flow quantities are normalized using the uniform incoming velocity $U_{\infty}$ and the diameter $D$ of the cylinder, which are the reference quantities used for the definition of the flow Reynolds number. Considering a frame of reference centered with the cylinder, with the $x$-axis aligned with the free-stream velocity and the $z$-axis with the cross-stream velocity, the dimensions of the computational domain used are : $-15 \leqslant x / D \leqslant 35$ and $-15 \leqslant z / D \leqslant$ 15. The velocity is imposed at the inflow $(x / D=-15)$, while stress-free conditions are applied on the lateral boundaries $(z / D= \pm 15)$ and on the outflow $(x / D=35)$.

The two-dimensional base flow is computed by Nek5000 using about 1500 spectral elements of degree $N_{p}=7$. This grid is also considered for the global stability analysis that allows to characterize the onset of the primary instability. In particular, the predicted value of the critical Reynolds number is equal to $R e_{c r} \simeq 46.2$, which compares very well with the literature. For instance, in Giannetti \& Luchini (2007) $R e_{c r}$ is estimated to be equal to $R e_{c r} \simeq 46.7$.

In order to show how the improved local stability analysis (with second-order correction effects included) performs in estimating the global stability properties, we consider the flow in supercritical conditions at $R e=50$. The corresponding unstable steady state is depicted in figure 1, where the streamline pattern around the cylinder is shown together with the $y$-vorticity. Performing the global stability analysis using Nek5000, the leading unstable global eigenvalue is $\omega_{G}(g l o b)=0.759+0.016 i$, well in agreement with Giannetti \& Luchini (2007). This value is also reported in table 1, put in comparison with other results from the literature, evaluated both with a global or a local stability approach.

When standard local analysis, i.e. without correction, is applied following the indications provided in section 2.2 , a region of absolute instability, where $\omega_{0, i}(X)>0$, is found in the near wake of the cylinder (not shown here for brevity), which extends in streamwise direction up to $X \simeq 3$.8. Since an analytical continuation of the local absolute frequency in the complex $X$-plane is required, the curve $\omega_{0}(X)$ is first interpolated with cubic splines to smooth out the numerical data and then analytically continued from the real $X$-axis into the complex space using an accurate Chebyshev rational polynomial approximation of order $N$. The saddle point is then identified using a Newton method, for values of $N$ ranging from 6 to 12 and no significant variations of the $X_{s}$ and $\omega_{s}$ are found. For instance, a comparison of the saddle point $X_{s}$ and $\omega_{s}$ is reported in the first two rows of table 2 , increasing the polynomial order $\mathrm{N}$ between 5 to 9 . As is clear, no significant discrepancies are shown varying the order of the polynomial extrapolation. In 


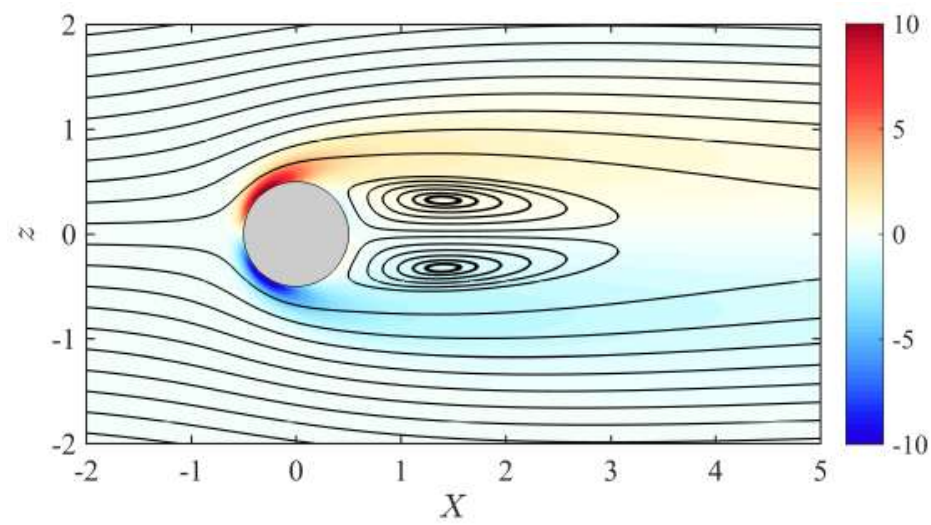

Figure 1. Vorticity (color) and streamlines of the flow past the circular cylinder at $R e=50$ (spacing in the $\mathrm{y}$-direction of $0.2 \mathrm{D}$ outside the separation bubble and of $0.04 \mathrm{D}$ in the recirculating region).

(a)
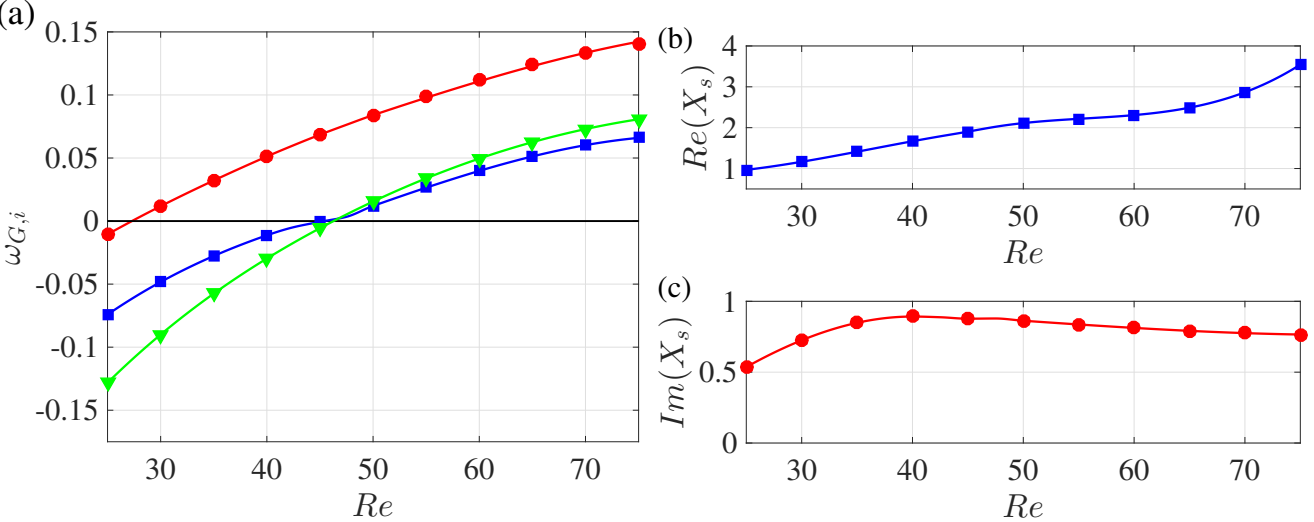

FiguRE 2. (a) Estimation of the $R e_{c r}$ for the primary wake instability of the flow past a circular cylinder. Green triangles: global analysis; red circles: local analysis not corrected; blue squares: local analysis with correction. Spatial position of the saddle point with Re: real (b) and imaginary (c) part of $X_{s}$, considering a rational function of $\mathrm{N}=7$.

\begin{tabular}{|c|c|c|c|}
\hline & Reference & $\omega_{g}$ & $\left|\omega_{r e f}-\omega\right| /\left|\omega_{r e f}\right|$ \\
\hline Global Analysis & $\begin{array}{l}\text { Present } \omega_{G}(\text { glob }) \equiv \omega_{\text {ref }} \\
\text { Giannetti \& Luchini }(2007)\end{array}$ & $\begin{array}{l}0.759+0.016 \mathrm{i} \\
0.750+0.013 \mathrm{i}\end{array}$ & $0 . \overline{4} \%$ \\
\hline Local analysis & $\begin{array}{l}\text { Pier }(2002) \\
\text { Juniper \& Pier (2015) } \\
\text { Present } \omega_{G, 0}(\text { loc }) \\
\text { Present } \omega_{G, 1}(\text { loc })\end{array}$ & $\begin{array}{l}0.785+0.091 \mathrm{i} \\
0.791+0.083 \mathrm{i} \\
0.750+0.084 \mathrm{i} \\
0.729+0.012 \mathrm{i}\end{array}$ & $\begin{array}{c}10.9 \% \\
10.4 \% \\
9.1 \% \\
3.1 \%\end{array}$ \\
\hline
\end{tabular}




\begin{tabular}{|c|c|c|c|}
\hline & $\mathrm{N}=5$ & $\mathrm{~N}=7$ & $\mathrm{~N}=9$ \\
\hline $\begin{array}{l}X_{s} \\
\omega_{G, 0}(l o c)=\omega_{s} \\
\omega_{G, 1}(l o c)=\omega_{s}+\epsilon \omega_{\epsilon}\end{array}$ & $\left|\begin{array}{l}+2.1130+0.8590 i \\
+0.7497+0.0842 i \\
+0.7298+0.0100 i\end{array}\right|$ & $\mid \begin{array}{l}+2.1109+0.8613 i \\
+0.7498+0.0842 i \\
+0.7289+0.0126 i\end{array}$ & $\mid \begin{array}{l}+2.1110+0.8628 i \\
+0.7498+0.0842 i \\
+0.7294+0.0113 i\end{array}$ \\
\hline $\begin{array}{l}\omega_{0 X X} \\
\omega_{k k} \\
k_{0 K}\end{array}$ & $\left|\begin{array}{c}+0.0271-0.0582 i \\
-0.1679-0.5686 i \\
+0.1100-0.0486 i\end{array}\right|$ & $\mid \begin{array}{l}+0.0261-0.0573 i \\
-0.1803-0.5496 i \\
+0.1113-0.0496 i\end{array}$ & $\mid \begin{array}{l}+0.0261-0.0567 i \\
-0.1664-0.5699 i \\
+0.1106-0.0489 i\end{array}$ \\
\hline
\end{tabular}

TABLE 2. Comparison of coefficients in equation $2.8\left(\omega_{0 X X}, \omega_{k k}, k_{0 K}\right)$ and complex global eigenvalues at different order of approximation $\left(\omega_{G, 0}(l o c), \omega_{G, 1}(l o c)\right)$ with increasing $N$, i.e. the order of rational function.

addition, following Juniper \& Pier (2015), these calculations have been repeated including progressively all points that satisfy the condition $\omega_{0, i}>\xi$, varying $\xi$ from 0.1 to -0.1 . This convergence test, whose results are not reported here for brevity, substantially confirmed the values reported in table 2 , with a discrepancy less than $2 \%$ for all of the cases.

The location of the complex saddle point is thus found at $\omega_{s}=\omega_{G, 0}(l o c)=0.750+$ $0.084 i, X_{s}=2.112+0.863 i$. These results are in agreement with Pier (2002); Juniper \& Pier (2015), as can be also evinced by table 1, thus validating the procedure and the numerical tools used for this study. The local approach predicts the global unstable eigenvalue within an error of $9.1 \%$. In this particular case, the error is larger for the complex part of the unstable eigenvalue, i.e. for the temporal growth factor. As a consequence, the standard local stability analysis estimates the critical Reynolds number $R e_{c r} \simeq 27.3$, which differs significantly from that one determined by a global stability analysis, with a percentage error of about $42 \%$.

The results of the local stability analysis can be corrected as detailed in section 2.3. First, the coefficients in equation 2.8 have to be evaluated at the complex saddle point $X_{s}$. Table 2 shows a comparison of coefficients appearing in 2.8 for increasing values of the order of rational function. Also for those quantities, a substantial independence of the results by changing the value of $\mathrm{N}$ is found. Using the equation (2.8), the approximated global eigenvalue is found equal to $\omega_{G, 1}(l o c)=0.729+0.012 i$, with a decreased error around $3 \%$ compared to that from the global stability analysis, as also reported in table 1. Here the accuracy on the predicted growth factor is largely improved and this leads to an accurate estimation of $R e_{c r}$, which is now estimated to be $R e_{c r} \simeq 45.2$, with an error of only $3.2 \%$ compared to that from the global stability analysis. Moreover, the complex eigenvalues $\omega_{G, 1}(l o c)$ evaluated with different order $\mathrm{N}$ of the rational function is reported in the third row in table 2. As a further assessment of the beneficial effects of the proposed correction, we report in Figure 2a the values of the global growth rate estimated by the local stability analysis with and without correction, i.e. $\omega_{G, 1}(l o c)$ and $\omega_{G, 0}(l o c)$ respectively, in comparison with the equivalent values estimated by the global stability analysis, for values of Reynolds number ranging from $\operatorname{Re}=25$ to $\operatorname{Re}=75$. The figure clearly shows that the applied correction systematically improves the predictions of the local stability analysis over the range of considered Reynolds numbers and, as already commented, significantly improves the prediction of the critical conditions for the onset of the wake instability. In addition, the position of the saddle point $X_{s}$ as a function of the Reynolds number is also reported in figures $2 b-c$. Since the length of the recirculation region in the near wake of the cylinder increased with the Reynolds number, 
(a)

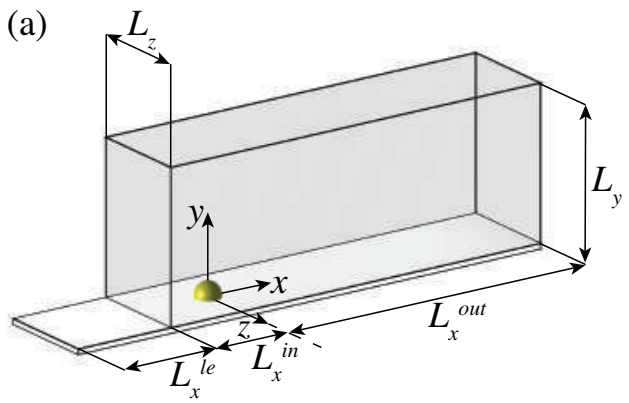

(b)

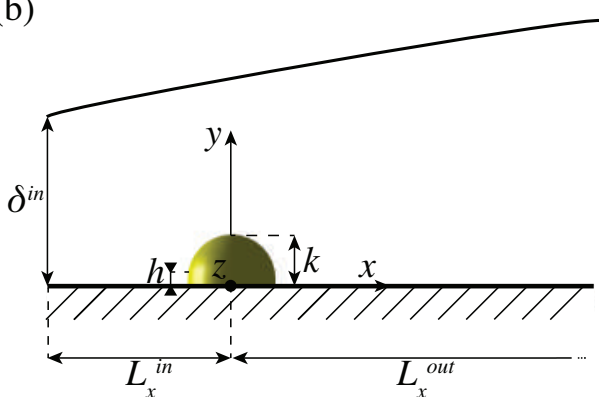

FiguRE 3. Flow past a wall-mounted hemispherical roughness: configuration and frame of reference (not in scale).

the saddle point moves slightly downstream, i.e. $\operatorname{Re}\left(X_{s}\right)$ increases. On the contrary, the distance of $X_{s}$ from the real $X$-axis, i.e. $\operatorname{Im}\left(X_{s}\right)$, remains substantially unchanged, at least for the considered range of Reynolds numbers.

\subsection{Boundary-layer flows past a hemispherical roughness element}

The first three-dimensional configuration here presented is the boundary-layer flows (BL) over a wall-mounted roughness element, sketched in figures 3a-b together with the considered Cartesian coordinate system. The roughness element is composed by a hemisphere of radius $R$ connected to the wall by a thin circular cylinder with the same radius and a height of $h=0.1 R$ (for further geometrical details see Citro et al. (2015)). For this case, the Reynolds number, $R e_{k}$, is based on the total height $k$ of the obstacle (see figure $3 \mathrm{~b}$ ) and the flow velocity $U_{k}$, i.e. the streamwise velocity at a distance $y=k$ from the wall that we would have inside the BL in the absence of the hemispherical obstacle at the same streamwise position. With reference to figure $3 \mathrm{a}$, the computational domain is characterized by $L_{x}^{i n}=7 R, L_{x}^{\text {out }}=30 R, L_{y}=8 R, L_{z}=10 R$ (labelled $M 1$ in Citro et al. (2015)) and the inherent mesh has about $9 \cdot 10^{3}$ spectral elements. Validation and convergence tests are reported in section $\S 2 \mathrm{D}$ in Citro et al. (2015). Concerning the boundary conditions for the DNS, homogeneous velocity $(\mathbf{u}=(0,0,0))$ is considered at the solid boundaries, a fully developed Blasius BL velocity profile is imposed at the inlet of the domain and, finally, outflow conditions, i.e. $p \mathbf{n}-R e^{-1} \nabla \mathbf{u} \cdot \mathbf{n}=0$, are imposed on the other boundaries.

For this flow configuration, the results from the WKBJ at higher order are presented and compared with those from the global analysis, considering the unsteady supercritical solution at $R e_{k}=450$. The wall-normal vorticity and the velocity streamlines of the base flow in the near-wake of the roughness element are reported in figure $5 \mathrm{a}$. At this value of $R e_{k}$, the flow is characterized by the shedding of periodic hairpin vortices inside the BL, with a Strouhal number $S t=\frac{\omega k}{2 \pi U_{k}} \simeq 0.16$ (for further details, see Citro et al. (2015)). As reported in figure $7 \mathrm{~b}$, the streamwise evolution of the absolute growth rate $\omega_{0, i}$ shows that the flow is absolutely unstable in the region $0.77<X<1.73$ This is reasonable in agreement with the dimensions of the recirculation region in the near wake of the hemispherical element, that extends in streamwise direction up to $X=2$ (see figure 7a).

Following the same procedure detailed in section $\S 4.1$ for the circular cylinder, the saddle point is found at $\omega_{s}=\omega_{G, 0}(l o c)=1.6322+0.1994 i, X_{s}=1.2923-0.047 i$ (figure $8 \mathrm{a}$ ). For a further verification, the location $\left(\omega_{s}, X_{s}\right)$ is also found by a graphical method seeking the cusp point in the $\omega_{0}$-plane. Referring to figure $5 \mathrm{~b}$, the streamwise evolution of $\omega_{0}$ is plotted at different value of the imaginary part of the complex X- 


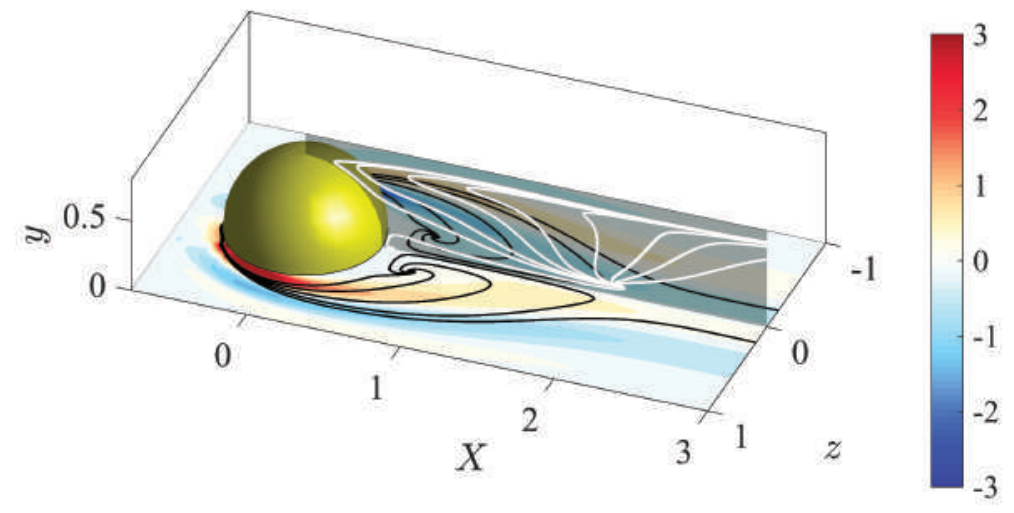

FiguRE 4. Streamlines of the flow past the hemispherical roughness element at $R e_{k}=450$ in the plane $y=0.03$ and $z=0$. Color contours depict the wall-normal vorticity in the plane $y=0.03$.
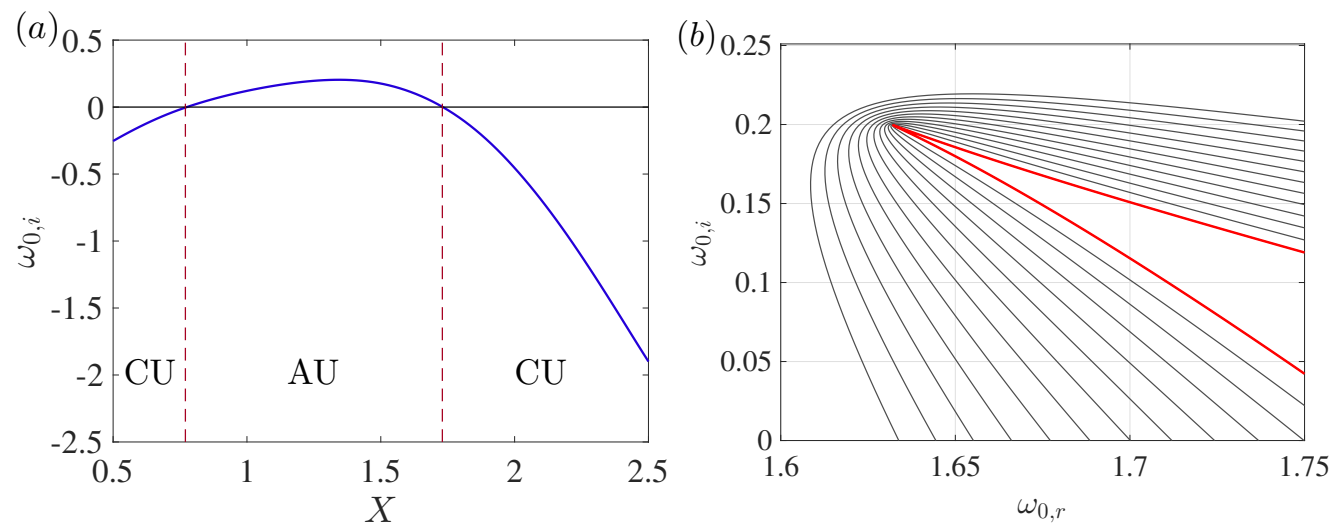

Figure 5. (a) Spatial evolution of the absolute growth rate $\omega_{0 i}$ (CU=convectively unstable flow, $\mathrm{AU}=$ absolutely unstable flow); (b) Evolution of the $\omega_{0}$ for different values of the complex $X$ with a 7th order rational function. The red line corresponds at the saddle point value, $\operatorname{Im}(X)=-0.047$ and the successive lines are evaluated at different $\operatorname{Im}(X)$, with intervals of -0.009 .

plane, $X_{s, i}$. The saddle point is then identified by the values for which a cusp formation is visible in the absolute frequency plane. The global analysis provides, for this flow configuration, a complex eigenfrequency of $\omega_{G}(g l o b)=1.858114+0.087148 i$ (Citro et al. 2015), which is slightly more stable than the local one. However, the over-prediction of the global growth-rate is a common characteristics of the local analysis, as already highlighted, for example, in Juniper et al. (2011). Moreover, the formula in equation 2.8 is here considered in order to obtain a better estimation of the global eigenvalue, taking into account the correction term $\omega_{\epsilon}$ in the WKBJ expansion at order $O\left(\epsilon^{1}\right)$. In particular, following the same extrapolation procedure used for the circular cylinder flow configuration presented in $\S 4.1$, the resulting complex global frequency is found equal to $\omega_{G, 1}(l o c)=1.6781+0.0376 i$. This result shows that, for this specific case, the correction procedure allows to obtain a better estimation of the global frequency, especially for the imaginary part of the eigenvalue. The correction coefficients appearing in equation 2.8, using $N=7$ as order of the rational function, are: $\omega_{0 X X}=1.8418-1.6684 i, \omega_{k k}=$ $0.0235-0.0715 i, k_{0 K}=1.6445-1.0907 i$. 


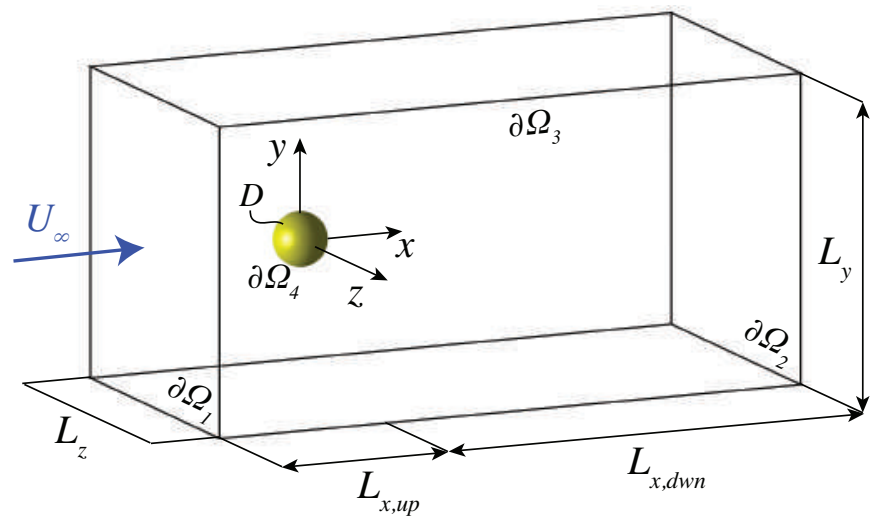

Figure 6. Flow past a spherical body: configuration and frame of reference (not in scale).

\subsection{Flow past a fixed sphere}

In this section, we detail the numerical results obtained in the case of a uniform stream that invests a fixed sphere of radius $R$. Figure 6 depicts the flow configuration and the frame of reference adopted in the present study. The reference quantities for the normalization of the flow and for the definition of the flow Reynolds number, $R e_{D}$, are the sphere diameter $D$ and the uniform streamwise velocity, $U_{\infty}$. The same flow configuration has been characterized by DNS and global stability analysis in Citro et al. (2016). In particular, we use here the same computational domain and discretization parameters adopted in Citro et al. (2016). The dimensions of the computational domain used for both the simulation and the global stability analisys are: $L_{x, u p}=12, L_{x, d w n}=35, L_{y}=24$, $L_{z}=24$; the mesh is made by approximately $13 \cdot 10^{3}$ spectral elements of degree $N_{p}=13$. We imposed a constant velocity profile $\boldsymbol{u}_{\boldsymbol{b}}=1 \boldsymbol{e}_{x}$ on the inlet boundary $\partial \Omega_{1}$ and on the lateral boundaries $\partial \Omega_{3}$ and a classical outflow condition is imposed on the outlet surface $\partial \Omega_{2}$. Validation and convergence tests leading to the adopted choice of the numerical discretization are detailed in Citro et al. (2016). Here, we focus our attention on the occurrence of the second bifurcation that arises at $R e_{D}=271.8$ and for $R e_{D}=275$ the flow is characterized by an asymmetric base flow and by the shedding of periodic vortical structures behind the bluff body, with a Strouhal number equal to $S t \simeq \frac{\omega D}{2 \pi U_{\infty}} \simeq 0.13$. An example of the steady unstable supercritical base flow is given in figure $7 \mathrm{a}$, where the modulus of the velocity field and the velocity streamlines are shown for a section $x-y$ plane located at $z=0.0$ (the mid-plane).

When the global stability analysis is performed on the flow field in figure 7a, a globally unstable mode is found with the corresponding complex eigenvalue $\omega_{G}(g l o b) \simeq 0.8092+$ $0.006 i$. The associated global mode (real part) is plotted in figures $9 \mathrm{e}-\mathrm{f}$.

When the classical local stability analysis, i.e considering only first order terms in the WKBJ expansion, is applied to the wake, a region of absolute instability is identified by inspecting where the absolute growth rate $\omega_{0, i}$ is positive. As shown in figure $7 \mathrm{~b}$, where the streamwise evolution of $\omega_{0, i}$ is depicted, the flow is absolutely unstable in the region $0.47<X<1.95$. The length of this region is comparable to the extension of the asymmetric recirculation bubble which extends in the streamwise direction from the top of the sphere, where separation first occurs, up to $X \simeq 2$ (see figure 7a).

As described in Sec. 2.2, continuing $\omega_{0}(X)$ in the complex $X$ plane, the eigenvalue of the globally unstable flow can be approximated at at the leading order $\epsilon$ by the eigenvalue of the saddle point $\left(\omega_{s}, X_{s}\right)$. The numerical procedure, already commented in the previuos 
(a)

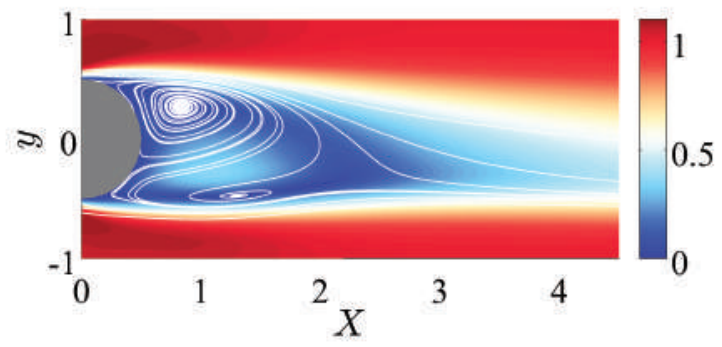

(b)

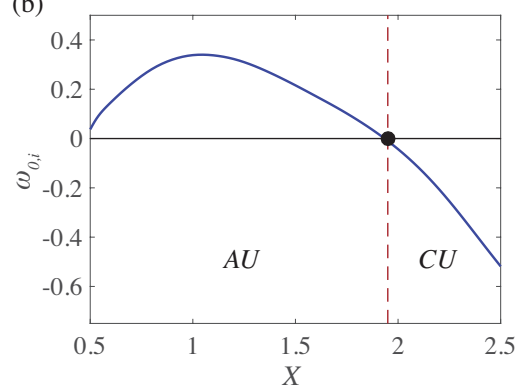

Figure 7. (a) Modulus of velocity (color) and streamlines of the flow past the fixed sphere at $R e_{D}=275$ in the midplane $(z=0.0)$; (b) Spatial evolution of the absolute growth rate $\omega_{0, i}$ $(\mathrm{CU}=$ convectively unstable flow, $\mathrm{AU}=$ absolutely unstable flow).
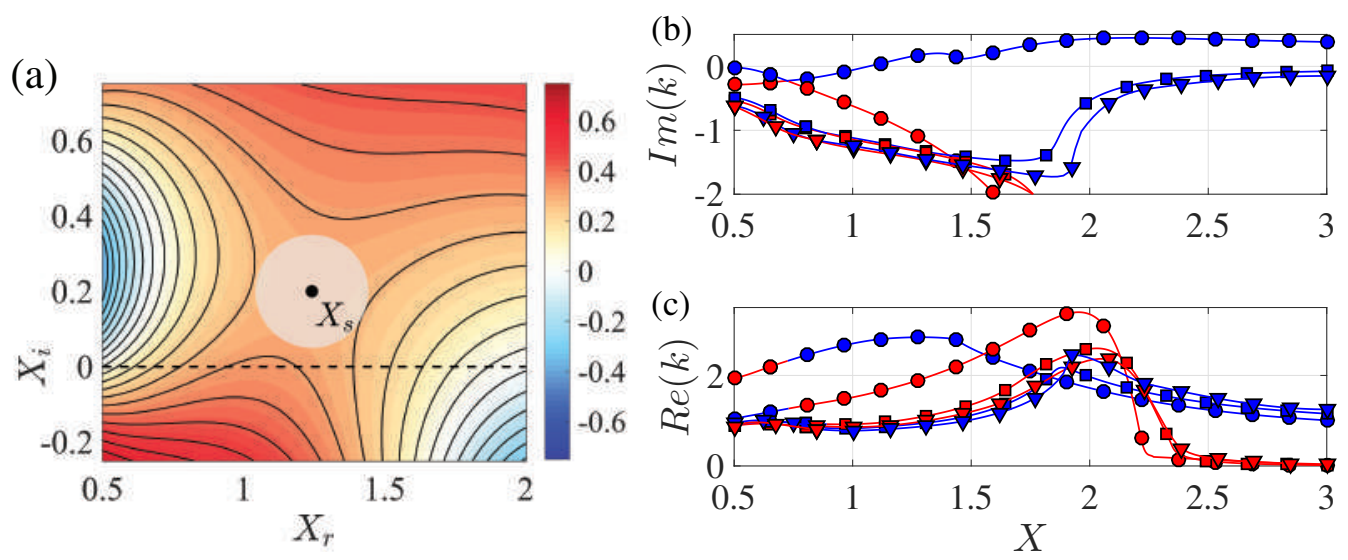

Figure 8. (a) $\omega_{0}, i$ map in the complex $X$ plane. (b) Streamwise evolution of the local wavenumbers $k^{+}$(blue lines) and $k^{-}$(red lines) obtained from the local dispersion relation using: imaginary components (b) and the real components (c) of the local wavenumber evaluated from the local analysis using $\omega=\omega_{G, 0}(l o c)(\bullet), \omega=\omega_{G, 1}(l o c)(\boldsymbol{\square})$ and $\omega=\omega_{G}(g l o b)(\boldsymbol{\nabla})$.

sections, allows to identify the saddle point at $\omega_{s}=\omega_{G, 0}(l o c)=0.6259+0.2711 i, X_{s}=$ $1.3394+0.2397 i$. This is shown in Figure $8 \mathrm{a}$, where $\omega_{0, i}$ is plotted in the complex $X$ plane. As in the case of the circular cylinder, the error $\left(\right.$ compared to $\left.\omega_{G}(g l o b) \simeq 0.8092+0.006 i\right)$ is distributed both on the frequency of the global mode and, in particular, on the growth rate, which is overestimated by a factor approximately equal to 45.2.

The evaluated global complex eigenvalue is then used in the local dispersion relation to evaluate the shape of the global mode. From equation 2.6 and following Juniper \& Pier (2015), the direct mode can be easily rebuilt. First, considering the saddle point complex frequency $\omega_{G, 0(l o c)}$, the spatial evolution of the complex wave-numbers $k^{\mp}$ and the corresponding local spatial eigenmode is evaluated from the dispersion relation for all the available $X$-section downstream the spherical body, using a streamwise spacing of $\Delta X=0.1$. The streamwise evolutions of the wavenumbers $k^{\mp}$ are shown in figures $8 \mathrm{~b}-\mathrm{c}$ with circle marks. The structure of the global eigenmode is then estimated using the equation 2.6. Figures 9a-b show the spatial evolution of the velocity streamwise component of the unstable mode The standard local analysis (WKBJ at $\mathrm{O}\left(\epsilon^{0}\right)$ as in Juniper et al. (2011)), therefore, provides useless results being the structure of the direct mode depicted in figure 9a-b very different from the corresponding global eigenmode depicted in figure 9e-f. Moreover, the relative error on the eigenvalue is about of $40 \%$. 
Local approach at $O\left(\varepsilon^{0}\right)$
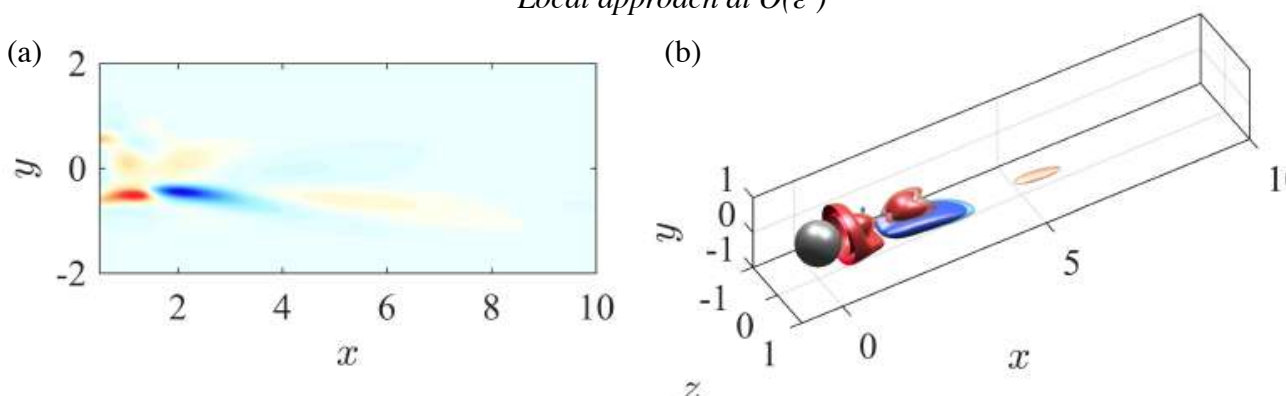

Local approach at $O\left(\varepsilon^{1}\right)$
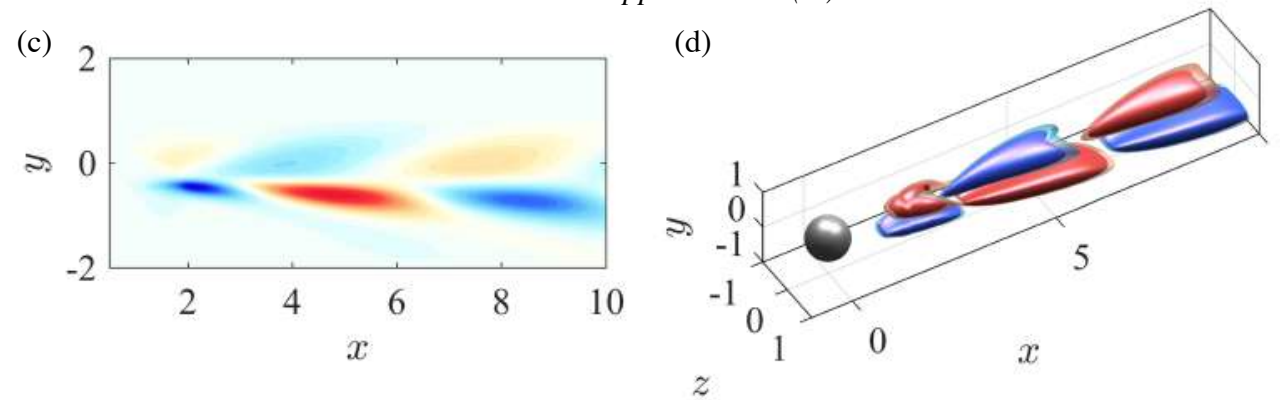

$3 D$ Global analysis
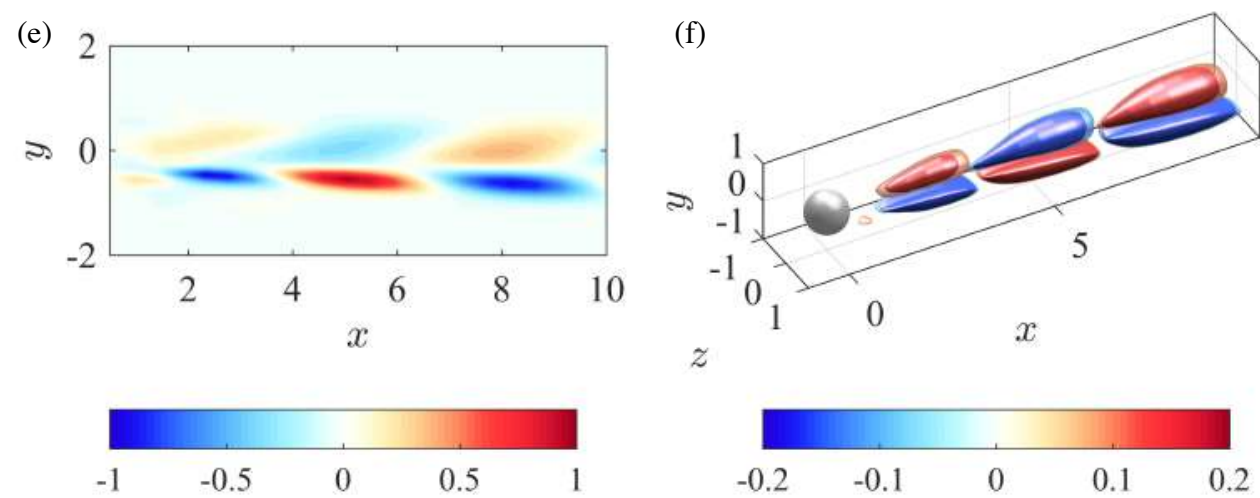

Figure 9. Comparison between the real part of the leading direct global mode calculated by using the standard local analysis (top), the improved local approach (middle) and the global stability analysis (bottom). (a,c,e) Contour plot of the streamwise velocity component $\hat{u}$ in the plane $(z=0.0)$. (b,d,f) Perspective view of the inherent isocontours.

As will be clear from the following paragraphs, in this case, the inclusion of higher order terms in the WKBJ expansion is needed to have acceptable results.

Repeating the correction procedure for the estimation of the global eigenfrequency at higher order (WKBJ at $\mathrm{O}\left(\epsilon^{1}\right)$ for this flow configuration, the corrected global eigenvalue is found to be $\omega_{G, 1(l o c)}=0.7460+0.0228 i$. The percentage error is now reduced to $8 \%$. For sake of completeness we provide the coefficients used in formula (2.8) to estimate the global eigenvalue with the correction at higher order: $\omega_{0 X X}=1.3135-0.0965 i$, $\omega_{k k}=-0.0334-0.1827 i$ and $k_{0 X}=-0.7553+0.9142 i$.

However, the most significant improvement is obtained on the estimation of the mode 
spatial evolution. As remarked above, non-parallel effects can be included by determining the slowly evolving function $A(X)$ with a solvability condition on the $O(\epsilon)$ equation of the WKBJ expansion. Another equivalent (up to higher order terms) approach is to compute the global mode shape using the corrected eigenvalue: $\omega_{G, 1(l o c)}$, i.e.

$$
\hat{\boldsymbol{q}}(x, y, z) \sim A^{\mp} \hat{\boldsymbol{q}}(y, z ; X) \exp \left[i \epsilon^{-1} \int_{X_{s}}^{X} k^{\mp}\left(\omega_{G, 1}(l o c) ; X^{\prime}\right) d X^{\prime}\right]
$$

where the local wavenumbers $k^{\mp}$ are now evaluated considering $\omega_{G, 1}(l o c)$ in the dispersion relation. Their streamwise evolutions are depicted in figures 8b-c (square marks) together with the branches $k^{\mp}$ evaluated forcing $\omega_{G}(g l o b)$ in the dispersion relation (triangle marks). ***

In a linear setting, the amplitude $A^{\mp}$ in 4.1 is undetermined, so that a normalization must be chosen when representing the eigenfunctions: here we use: $\max |\hat{u}(X, y, z)|=1$. Figures 9c-d show the spatial distribution of the velocity streamwise component of the unstable mode retrieved using the corrected eigenvalue $\omega_{G, 1}(l o c)$. All considered cases are depicted in figure 9, where representative isosurfaces of the mode with the same amplitude and phase normalization are shown. Finally, we can note the excellent agreement between the rebuilt spatial structure and the corresponding mode shape obtained by using a full $3 \mathrm{D}$ global stability analysis.

\section{Summary}

In this paper, we show that classical local spatio-temporal stability analysis of weakly non-parallel flow can be straightforwardly applied to fully 3D base flows. Moreover, we show that the inclusion of higher-order terms in the WKBJ analysis is a crucial ingredient in order to obtain results which compare quantitatively well with those provided at definitely higher computational costs through global stability analysis. In particular, in this paper, we first consider the flow past a circular cylinder where the correction term provides a significant improvement of the results in comparison with those obtained by a global stability analysis. For instance, previous numerical studies (Giannetti \& Luchini 2007 ) showed that the simple local analysis at order $\mathrm{O}\left(\epsilon^{0}\right)$ fails to predict the critical Reynolds number for such flow. We show that the inclusion of $\mathrm{O}\left(\epsilon^{1}\right)$ terms reduces the relative error from $42 \%$ to $3.2 \%$.

Second, we show that an equivalent improvement in the prediction of the global stability properties is obtained for two three-dimensional configurations, i.e. the boundary layer flow past a wall-mounted hemispherical obstacle and the secondary instability of the sphere wake. Indeed, the complex eigenvalues evaluated by the current improved local approach are in good agreement with the one obtained by the global stability analysis. For the sphere case, the inclusion of $O(\epsilon)$ terms in the WKBJ expansion not only considerably reduces the eigenvalue error but also provide a drastic improvement to the shape reconstruction of the global mode, curing in this way the large discrepancies between the local and the global results. The present study confirms that a reasonable good estimation of the global mode characteristics can be obtained just relying on a local stability approach, with a computational cost of orders of magnitude lower than that necessary for a global analysis.

\section{REFERENCES}

Bender, C. M. \& Orszag, S. A. 1978 Advanced Mathematical Methods for Scientists and Engineers. McGraw Hill. 
Bers, A. 1983 Space-time evolution of plasma instabilities-absolute and convective. In Handbook of Plasma Physics, vol.1, pp 451-517. McGraw Hill.

BRiggs, J. 1964 Electron-stream interaction with plasmas. M.I.T. Press pp. xii +187.

Chomaz, J. M., Huerre, P. \& Redekopp, L. G. 1988 Bifurcations to local and global modes in spatially developing flows. Phys. Rev. Lett. 60, 25-28.

Citro, V., Giannetti, F., Luchini, P. \& Auteri, F. 2015 Global stability and sensitivity analysis of boundary-layer flows past a hemispherical roughness element. Phys. Fluids 27, 084110 .

Citro, V., Tchoufag, J., Fabre, D., Giannetti, F. \& Luchini, P. 2016 Linear stability and weakly nonlinear analysis of the flow past rotating spheres. Submitted to Journal of Fluid Mechanics .

Cooper, A. J. \& Crighton, D. G. 2000 Global modes and superdirective acoustic radiation in low-speed axisymmetrics jets. Eur. J. Mech. B Fluid 19, 559-574.

Giannetti, F. \& Luchini, P. 2007 Structural sensitivity of the first instability of the cylinder wake. Journal of Fluid Mechanics 581, 167-197.

Huerre, P. \& Monkewitz, P. A. 1985 Absolute and convective instabilities in free shear layers. Journal of Fluid Mechanics 159, 151-168.

Huerre, P. \& Monkewitz, P. A. 1990 Local and global instabilities in spatially developing flows. Annual Review of Fluid Mechanics 22, 473-537.

Huerre, P. \& Rossi, M. 1998 Hydrodynamics Instabilities in open flows. in Hydrodynamics and Nonlinear Instabilities. Cambridge University Press.

Juniper, M.P., Tammisola, O. \& Lundell, F. 2011 The local and global stability of confined planar wakes at intermediate reynolds number. Journal of Fluid Mechanics 686, 218-238.

Juniper, M. P. \& PIER, B. 2015 The structural sensitivity of open shear flows calculated with a local stability analysis. Eur. J. Mech. B Fluid 49, 426-437.

Monkewitz, P. A., Huerre, P. \& Chomaz, J. M. 1993 Global linear stability analysis of weakly non-parallel shear flows. Journal of Fluid Mechanics 251, 1-20.

PiER, B. 2002 On the frequency selection of finite-amplitude vortex shedding in the cylinder wake. Journal of Fluid Mechanics 458, 407-417.

PiER, B. 2008 Local and global instabilities in the wake of a sphere. Journal of Fluid Mechanics 603, 39-61.

Siconolfi, L., Camarri, S. \& Fransson, J. H. M. 2015 Stability analysis of boundary layers controlled by miniature vortex generators. Journal of Fluid Mechanics 784, 596-618.

Theofilis, VAssilios 2011 Global linear instability. Annual Review of Fluid Mechanics 43, 319-352. 